\title{
Constraining Dark Matter hypothesis through observations of Dwarf Spheroidal galaxies with the Fermi-LAT detector.
}

\author{
J. Cohen-Tanugi, C. Farnier and E. Nuss* on behalf of the Fermi-LAT collaboration \\ L.P.T.A. Université Montpellier 2, CNRS/IN2P3 - Montpellier France \\ E-mail: eric.nusselpta.univ-montp2.fr
}

\begin{abstract}
Measurement of $\gamma$-rays from dark matter annihilations is perhaps one of the most challenging tasks for future $\gamma$-ray observatories, such as the Fermi Gamma-Ray Space Telescope (in short "Fermi"). Fermi, launched on June 11th 2008, is the next generation satellite for high-energy $\gamma$ ray astronomy and the Fermi Large Area Telescope (LAT), Fermi main instrument, has excellent potential to either discover or to constrain a dark matter signal.

Dwarf spheroidal galaxies, the largest clumps predicted by the cold dark matter scenario, are attractive targets for indirect search of dark matter because they are amongst the most extreme dark matter dominated environments.

We present an overview of the Fermi LAT Dark Matter and New Physics Working Group efforts in the study of the LAT capability to detect a $\gamma$-ray flux coming from WIMP pair annihilations in dwarf spheroidal galaxies.
\end{abstract}

Identification of dark matter 2008

August 18-22, 2008

Stockholm, Sweden

${ }^{*}$ Speaker. 


\section{Introduction}

The Fermi Gamma-Ray Space Telescope (in short "Fermi") [1, 2], which is part of the NASA's office of Space and Science strategic plan, is a next generation satellite-based observatory designed to explore the high-energy $\gamma$-ray sky ${ }^{1}$. This mission, realized as a close collaboration between the astrophysics and particle physics communities, (including institutions in the USA, Japan, France, Germany, Italy and Sweden) has been successfully launched on June 11th 2008 and is being operating in nominal science configuration. The main instrument on Fermi, the Large Area Telescope (LAT) takes much of its basic design concept from its predecessor EGRET but the energy range (over more than four energy decades $20 \mathrm{MeV}-300 \mathrm{GeV}$ ), field-of-view ( $>2 \mathrm{sr}$ ), angular resolution and large effective area will provide the LAT with unprecedented sensitivity and resolution, filling the gap between the previous generation of $\gamma$-ray space missions and the ground based Cerenkov detectors. This improvement should allow the LAT to detect several thousands of new high-energy sources, shed light on many issues left open by EGRET and provide excellent high-energy $\gamma$-ray observations for Dark-Matter searches. The Fermi-LAT collaboration will explore many complementary searches for DM signal and several studies have been performed to determine the sensitivity of Fermi to DM annihilation signal [3, 4, 5, 6]. We will focus here on the Fermi-LAT capabilities to constrain DM hypothesis through observations of dwarf spheroidal galaxies (dSphs).

\section{Sensitivity to Dark Matter annihilations from Dwarf Spheroidal galaxies with the Fermi-LAT detector.}

While dark matter has not been directly detected in laboratory experiments, there is a wealth of experimental evidences and arguments for a Cold Dark Matter (CDM) component of the matter density of the Universe. The question of what constitutes this CDM has been a subject of special interest to high-energy physicists, astrophysicists and cosmologists for many years and is one of the most fascinating and intriguing issues in present day cosmology. Virtually all proposed CDM candidates require physics beyond the standard model of particle physics. One of the most widely studied model is supersymmetric extensions of the standard model which provides a natural candidate for CDM in the form of a stable uncharged Majorana fermion, the Neutralino. The mutual annihilation of this Weakly Interacting Massive Particle (WIMP) would yield, among a few other indirect signatures like energetic neutrinos, antiprotons or positrons, many high energy $\gamma$-rays $(\geq 1$ $\mathrm{GeV}$ ) that can be well measured in the Fermi LAT and may give rise to a signal in $\gamma$-ray spectra from many cosmic sources.

Results from N-body simulations on test particles with only gravitational interactions [7] shows that WIMPs are expected to form a large amount of substructure. The dwarf spheroidal galaxies, the largest clumps predicted by the CDM scenario, are ideal laboratories for indirect search of DM because astrophysical backgrounds and baryon-dark matter interactions are expected not to play a major role in the DM distribution. The uncertainties in the DM distribution can be better quantified and understood. Furthermore, the mass-to-light ratios in dSphs can be very large, showing that they are largely DM dominated systems. For example, the mass-to-light ratio of Draco is $\sim 250$ in Solar units [8], while it is $\sim 100$ [9] for the Sagittarius dwarf. In addition, dSphs are expected

\footnotetext{
${ }^{1}$ For more details, see the Fermi website at: http://glast.gsfc.nasa.gov/
} 
to be relatively free of intrinsic $\gamma$-ray emission from other astrophysical sources, thus eliminating contaminating background that may hinder the interpretation of any detection. Their relative proximity and high Galactic longitude and latitude makes some of them ideal for high signal-to-noise detection.

During the first year, Fermi will operate in an all-sky survey mode leading to an uniform all-sky coverage. As an illustration of the Fermi-LAT capabilities to detect DM $\gamma$-ray signal from dSph galaxies, we computed the $3 \sigma$ sensitivity for Sagittarius and Draco dSphs for 5 years operation. The results for these two dwarfs is well representative of the LAT sensitivity to dSph DM signal as Sagittarius is an extended source located near the galactic plane and Draco, seen by the LAT as a point like source, is located at higher latitude which make the sensitivity to this source less dependent on the Galactic diffuse background modelling. Discovered in 1994, Sagittarius is one of the $\sim 22 \mathrm{dSphs}$ orbiting our own Galaxy. The core of Sagittarius dwarf is located at $l=5.61^{\circ}$ and $b=-14.08^{\circ}$ in Galactic coordinates at a distance of about $24 k p c$ from the Sun which make Sagittarius one of the nearest satellite of the Milky Way. The core of Draco dwarf, discovered in 1954, is located at $l=85.37^{\circ}$ and $b=34.72^{\circ}$ at a distance of about $80.1 \mathrm{kpc}$. As most dSphs do not contain gas, the structure of the dark halos must be inferred from stellar motion. For this study we used the Moore cusped halo profile derived for Sagittarius and Draco in [10]. Note that a more conservative NFW profile would lead to decrease the senstivity by about a factor of ten. For EGRET compatibility, we required here that the total integrated flux does not exceed the value extracted from [11].

To compute the Fermi-LAT sensitivity to DM, we used the observation simulation tool gt obss im from the ScienceTools software package [12], which is being developed jointly by the LAT collaboration and the GLAST Science Support Center. The observation simulation tool allows users to define Celestial $\gamma$-ray sources with arbitrary spectral properties. For a generic model of WIMPs annihilating into $b \bar{b}$, we generated the WIMP annihilation differential fluxes above $1 \mathrm{GeV}$ for a scan in the plane defined by the WIMP mass $m_{\text {wimp }}$ and the self annihilating cross section $\langle\sigma v\rangle$. An example of the expected count map in the Sagittarius dwarf region for $m_{\text {wimp }}=86 \mathrm{GeV}$ and $<\sigma v\rangle=310^{-26} \mathrm{~cm}^{3} \mathrm{~s}^{-1}$ is shown in Fig.1 (Left). In a first step, after computing the expected flux, we performed a standard $\chi^{2}$ statistical analysis for every couple of values $\left(m_{\text {wimp }},\langle\sigma v>)\right.$ to see whether Fermi is able to disentangle the WIMP contribution among the standard astrophysical background as described in [3]. The result for Sagittarius dwarf is given at a $3 \sigma$ confidence level and shown in Fig.1 (Right).

Additionally to the observation simulation tool, the ScienceTools package includes source analysis tool gtlike which uses maximum likelihood to fit source spectral parameters such as flux and power-law spectral index though more complex spectral models are available. Since the detected counts for sources near the detection limit will be fairly low, gt l i ke calculates a likelihood function based on the Poisson probability using the source model folded through the LAT instrument response functions [13] to provide the expected model counts. As an example, the Fig 2 (Left) shows the resulting fit for the model used in Fig.1 (Left). In a second step, we computed the test statistic (TS) as the maximum likelihood difference between background only and background plus signal, (where the free fit parameter is the signal normalization) to extract the $3 \sigma$ sensitivity as a function of the WIMP mass and annihilation cross-section. The blue lines which have been superimposed to the $\chi^{2}$ sensitivity of Fig.1 (Right) have been obtained from likelihood analysis applied 

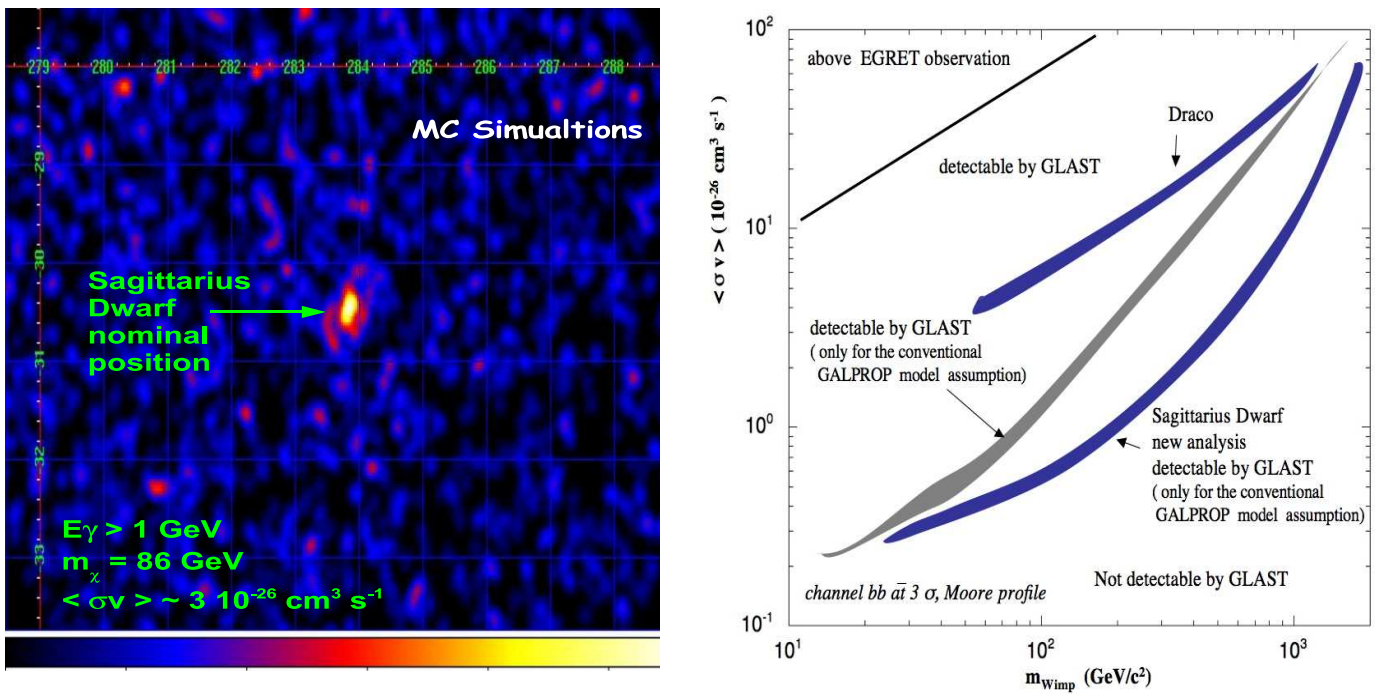

Figure 1: Left : Expected count map $\left(E_{\gamma}>1 \mathrm{GeV}\right)$ in the Sagittarius dwarf region for $m_{\text {wimp }}=86 \mathrm{GeV}$ and $<\sigma v\rangle=310^{-26} \mathrm{~cm}^{3} \mathrm{~s}^{-1}$. Right : Fermi sensitivity in the $\left(m_{\text {wimp }},\langle\sigma v\rangle\right)$ plan from $\chi^{2}$ analysis (grey line) for a $b \bar{b}$ annihilation signal from the Sagittarius dwarf. The upper part of the phase space is already excluded by the EGRET data and the lower part is not detectable by Fermi [3]. Blue lines, corresponding to a likelihood analysis for Sagitttarius and Draco dSphs (see text), has been superimposed.

to Draco (upper line) and Sagittarius dwarfs (lower line) simulated data. Regarding to the $\chi^{2}$ analysis [3], the likelihood analysis lead to an improved sensitivity of a factor 2 to 4 .

The Fig 2 (Right) shows how our sensitivity can be compared with the mSUGRA and MSSM models in the $\left(m_{\text {wimp }},<\sigma v>\right)$ plan. All plotted models are consistent with all accelerator constraints and WMAP data.

\section{Conclusions and outlook}

Fermi is in routine science operations since August 11th, 2008. In this proceeding we summarized how the Fermi-LAT observatory could help to disentangle the DM puzzle through the observations of dwarf spheroidal galaxies. Even if the amount of all-sky scanning data required for obtaining significant DM signal is estimated to be at least 1 year, we have shown that, in the scenarios that have been considered, the results are promising [3]. However, the establishment of a detection will require thorough understanding of the involved backgrounds and possibly collaborative efforts with ground based Air Cherenkov telescope experiments.

\section{References}

[1] W. B. Atwood [GLAST Collaboration], Nucl. Instrum. Meth. A 342, 302 (1994)

[2] P. F. Michelson [GLAST-LAT Collaboration], AIP Conf. Proc. 921, 8 (2007)

[3] E. A. Baltz et al., JCAP 0807 (2008)

[4] J.Cohen-Tanugi, these proceedings. 

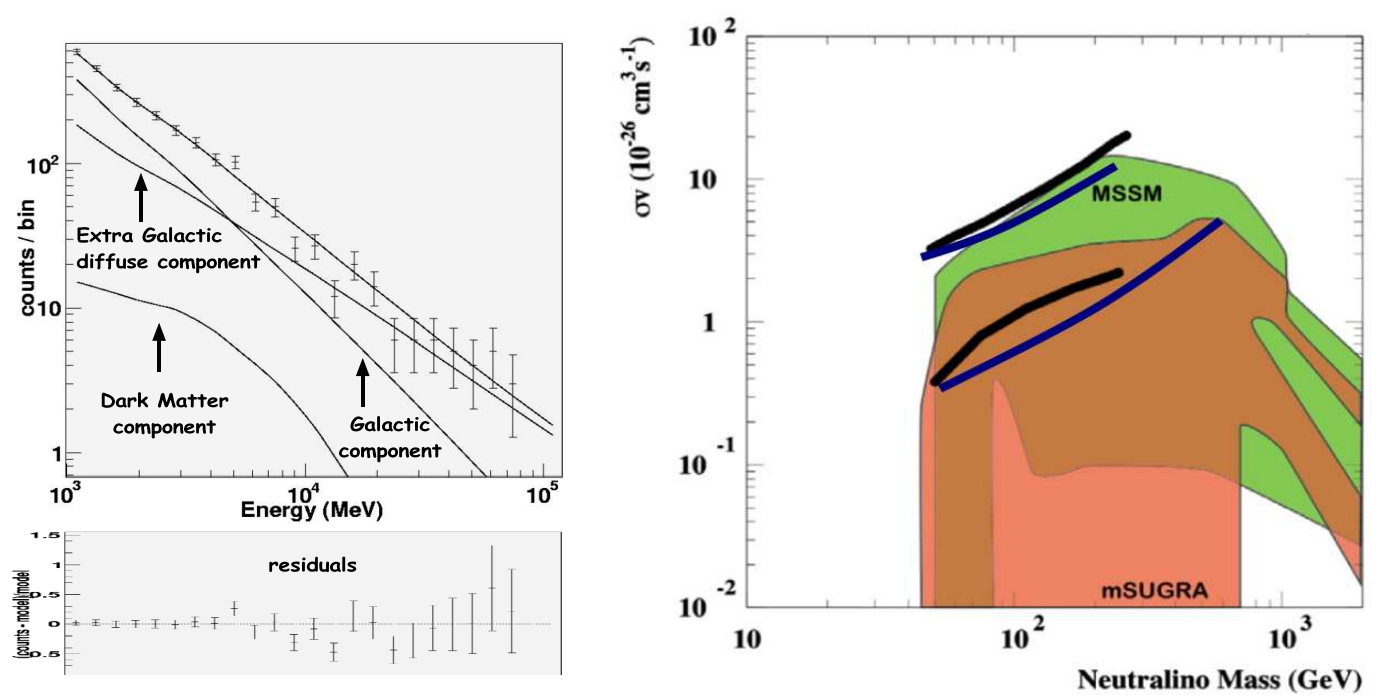

Figure 2: Left : Resulting fit from likelihood analysis of the model shown in Fig.1 (Left). Right : MSSM and mSUGRA models in the $\left(m_{\text {wimp }},\langle\sigma v>)\right.$ plane. The dark lines indicate the Fermi $5 \sigma$ sensitivity for a DM search in the Galactic Center (upper line) and in the galactic halo (lower line) [3]. The blue lines correspond to the $3 \sigma$ sensitivity obtained from likelihood analysis on Draco (upper line) and Sagittarius (lower line) dSphs simulated data.

[5] V.Vitale, these proceedings.

[6] T.Ylinen, these proceedings.

[7] V. Springel et al. Nature, 435, 629 (2005)

[8] J. T. Kleyna, M. I. Wilkinson, N. W. Evans and G. Gilmore Astrophys. J. 563, L115 (2001)

[9] R. A. Ibata, R. F. G. Wyse, G. Gilmore, M. J. Irwin, N. B. Suntzeff, Astron. J. 113, 634 (1997)

[10] N. W. Evans, F. Ferrer and S. Sarkar, Phys. Rev. D 69, 123501 (2004)

[11] R. C. Hartman et al. [EGRET Collaboration], Astrophys. J. Suppl. 123 (1999) 79

[12] http://glast.gsfc.nasa.gov/ssc/data/

[13] http:

//www-glast.slac.stanford.edu/software/IS/glast_lat_performance.htm 